\section{SPECIAL SUBSCRIBERS} natuTE SUBME ON-APPRK OFFER-EXAMS WITHOUT RISK

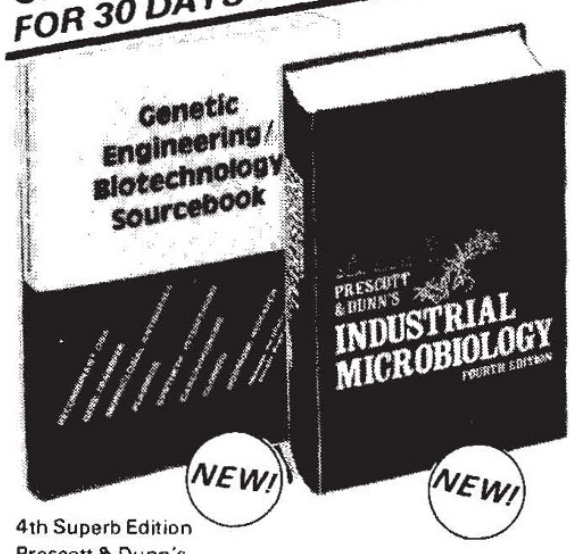

Prescatt 2 Dunn's

\section{Industrial Microbiology}

Edited by Gerald Reed

Vice President, Amber Laboratories USA

The up-to-date guide and reference tool to industrial technology and applications in the microbiology of foods and food ingredients - with a special section on biological production of fuel alcohol.

Macmillan Hardcover $896 \mathrm{pp} \quad £ 42.50$
ISBN 3333363051982

\section{Genetic Engineering/}

\section{Biotechnology Sourcebook}

Never before published! Profiles of 1,529 recent and ongoing genetic engineering/biotechnology research projects funded by 122 US government and non-profit organizations.

Covers research affecting: Pharmaceuticals $\star$ Medicine and Diagnosis $\star$ Agriculture $\star$ Food Processing $\star$ Energy $\star$ Chemicals.

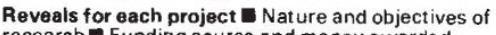
research Funding source and money awarded
- Names and addresses of individual researchers.

Special Yellow Pages reference section - tells you at a glance who's researching what, where, when and how.

Macmillan Softcover $350 \mathrm{pp} \quad \mathbf{f 5 2 . 5 0}$

ISBN 03333414811982

Place your on-approval order today!

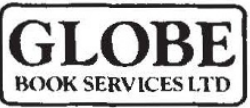

Canada Road, Byfleet, Surrey KT14 7JL, England. Tel: Byfleet (09323) 40397

A MEMBER COMPANY OF MACMILLAN PUBLISHERS LTD.

EXAMINE ON 30 DAYS APPROVAL

To: lan Jacobs, Globe Book Services Ltd., FREEPOST, Canada Road, Byfleet, Surrey KT14 7BR. England. (Please tick books required)

Please send me _- copies of $\square$ Genetic Engineering/Biotechnology Sourcebook at 152.50 each plus $P$ \& $(U K\{2$. Overseas $f 3$.) and $/ 0$

copies of $\square$ Industrial Microbiology at $£ 42 . \overline{50 \text { each }}$ plus P\&P (UK $\{2$. Overseas $f 3$ ) on 30 days approval. If am not completely satisfied. I mayr return thevolume(s) within that period for a full refund.

Tick preferred method of payment

$\square$ I enclose cheque for $f$

$\square$ Charge my credit card $f$

$\square$ Visa $\square$ Access $\square$ American Express $\square$ Diners

Card No.

$\square$ Expiry date

Signature

(Orders cannot be accepted without a signature)

Name - (PLEASE PRINT)

Address

(PLEASE PRINT)

Allow 28 days for delivery

Postcode

POST TODAY - NO STAMP NEEDED

\title{
The protocol for arranging nature
}

\section{Michael B. Usher}

Multivariate Analysis in Community Ecology. By Hugh G. Gauch Jr. Pp.298. Hbk ISBN 0-521-23820-X; pbk ISBN 0-521-28240-3. (Cambridge University Press: 1982.) Hbk £20, \$37.50; pbk £7.95, $\$ 14.95$.

ECOLOGISTS collect multivariate data. Very rapidly the number of observations, each being the occurrence or abundance of one of a large number of species in one of a large number of quadrats, baffles the observer, who needs some kind of protocol for arranging and viewing the data. Multivariate analyses provide that protocol, but the diversity of possible methodologies can confuse all but the most numerate of ecologists.

In this new book, Hugh Gauch attempts to explain the methodology of multivariate analysis without delving into the mathematical or computational complexities. The introductory chapter deals with hand sorting: the sceptic only needs to look at Table 1.2 with 50 species recorded from 25 quadrats to see how even a small data set can look incomprehensible until it is re-arranged, as in Table 1.3.

Gauch makes the point that more objective methods for arranging all of the individual observations are required, and he devotes the remainder of the book to discussion of the three basic approaches to sorting ecological data of this type - direct gradient analysis, ordination and classification. Although the structure of each chapter differs, generally the rationale of the approach is described, the methods are explained in words rather than in mathematical symbols, a number of examples are quoted (illustrations lead to a geometrical interpretation of the results), some of the known problems are described, and finally there is a broad evaluation. It is these chapters which give the book its value.

There are two drawbacks, however, that prevent this being a complete textbook. First, if anyone actually wishes to undertake an analysis they will have to turn to another book for the recipe. True, there is a two-page appendix included on computer programs, but this is too brief to be a usable consumer's guide. Second, the text is very Cornell-orientated - perhaps not surprisingly, since Gauch is closely associated with the group which worked with the late R. H. Whittaker at that university. An example is the "horseshoe" problem, where their DECORANA method of tackling it is described but others are not considered (though to be fair the extensive catalogue of applications in Chapter 6 partly overcomes this problem).

Nonetheless, the clear explanation and the comparisons between the various approaches make the book a suitable text for the student or researcher who wishes - or has - to understand this field of quantitative ecology. As the author in general cleverly avoids the mathematical complications, the apparent simplicity may be beguiling. But I still plan to use this book as a background text when teaching multivariate methods.

Michael B. Usher is a Senior Lecturer in the Department of Biology at the University of York.

\section{Teaching machines}

\section{Margaret Boden}

Intelligent Tutoring Systems. Edited by D. Sleeman and J.S. Brown. Pp.345. Hbk ISBN 0-12-648680-8. (Academic: 1982.) $£ 19.20, \$ 39.50$.

INTELLIGENT tutoring systems (ITSs) are programmed teaching-aids grounded in artificial intelligence (AI). They differ radically from familiar types of computerassisted-instruction (CAl). Many of the latter merely present repetitive "drill-andpractice" exercises; others can vary their response with the student's level of understanding by means of branched programs with predefined choice-points, but only to a very limited degree. ITSs are considerably more flexible, and better able to adapt to the idiosyncracies of a student's thinking.

This flexibility derives from the fact that ITSs contain complex computational models of students' knowledge and thinking. These control the questions and responses that an ITS presents to the student, and generate its diagnoses of students' mistakes. Unlike traditional CAI, the emphasis of ITSs is less on facts than on ways of thinking about a problemdomain; they function not so much by presenting " correct" examples of solutions as by providing relevant hints or questions, and - significantly - by diagnosing the thought-strategies behind the student's mistakes so as to guide the tutorial response.

Such diagnosis requires an understanding (in explicit, programmed, form) of the variations in cognitive structure and inference that underlie different sorts of (non-random) mistake. Computer science has a word for such mistakes - "bugs". A bug is a precisely definable and relatively systematic erroneous variation of a correct procedure (this definition, though, optimistically implies an underlying correctness). Much work on ITSs is focused on identifying and using bugs in the student's thinking.

For example, DEBUGGY is a program that is better than human beings at diag- 